\title{
Aortic Stiffness and Diastolic Dysfunction in Sprague Dawley Rats Consuming Short-Term Fructose Plus High Salt Diet
}

This article was published in the following Dove Press journal: Integrated Blood Pressure Control

\author{
Dragana Komnenov ${ }^{1}$ \\ Peter E Levanovich ${ }^{2}$ \\ Natalia Perecki (i) \\ Charles S Chung (D) ${ }^{2}$ \\ Noreen F Rossi ${ }^{1-3}$ \\ 'Department of Internal Medicine, \\ Division of Nephrology, Wayne State \\ University School of Medicine, Detroit, \\ MI, USA; ${ }^{2}$ Department of Physiology, \\ Wayne State University School of Medicine, \\ Detroit, MI, USA; ${ }^{3}$ Department of Research \\ and Development, John D. Dingell Veterans \\ Affairs Medical Center, Detroit, MI, USA
}

Correspondence: Noreen F Rossi Department of Internal Medicine, Division of Nephrology, Wayne State University School of Medicine, 4160 John R Street \#908, Detroit, MI 4820I, USA Email nrossi@wayne.edu
Introduction: High fructose and salt consumption continues to be prevalent in western society. Existing studies show that a rat model reflecting a diet of fructose and salt consumed by the upper 20th percentile of the human population results in salt-sensitive hypertension mitigated by treatment with an antioxidant. We hypothesized that dietary fructose, rather than glucose, combined with high salt leads to aortic stiffening and decreased renal artery compliance. We also expect that daily supplementation with the antioxidant, 4-hydroxy2,2,6,6-tetramethylpiperidine-1-oxyl (+T; Tempol), will ameliorate the increase in mean arterial pressure (MAP) and vascular changes.

Methods: Male Sprague Dawley rats were studied with either $20 \%$ fructose or $20 \%$ glucose in the drinking water and normal salt $(0.4 \%)$ or high salt $(4 \%)$ in the chow resulting in four dietary groups: fructose normal Fru+NS or high salt (Fru+HS) or glucose with normal (Glu $+\mathrm{NS})$ or high salt $(\mathrm{Glu}+\mathrm{HS})$. Tempol $(+\mathrm{T})$ was added to the drinking water in half of the rats in each group for 3 weeks.

Results: MAP was significantly elevated and the glucose:insulin ratio was depressed in the Fru + HS. Both parameters were normalized in Fru+HS+T. Plasma renin activity (PRA) and kidney tissue angiotensin II (Ang II) were not suppressed in the high salt groups. Pulse wave velocity (PWV), radial ascending strain, and distensibility coefficient of the ascending aorta were significantly decreased in Fru+HS rats and improved in the Fru+HS+T rats. No differences occurred in left ventricular systolic function, but the ratio of early (E) to late (A) transmitral filling velocities was decreased and renal resistive index (RRI) was higher in Fru+HS rats; antioxidant treatment did not change these indices.

Discussion: Thus, short-term consumption of high fructose plus high salt diet by rats results in modest hypertension, insulin resistance, diminished aortic and renal artery compliance, and left ventricular diastolic dysfunction. Antioxidant treatment ameliorates the blood pressure, insulin resistance and aortic stiffness, but not renal artery stiffness and left ventricular diastolic dysfunction.

Keywords: angiotensin II, hypertension, insulin resistance, pulse wave velocity, renin

\section{Plain Language Summary}

High fructose and high salt consumption is very common in western society. Rats were given a diet that mimics the amounts of fructose and salt eaten by one-fifth of the human population and were compared with rats eating fructose and normal salt diet or rats fed a diet that provided the same number of sugar calories in the form of glucose and either high or normal salt diet. Even after a short 3-week period, the rats on the combined high fructose and high salt diet had higher blood pressure than any of the other groups. Rats fed high 
fructose and high salt diet also developed stiffening of their major large arteries, including the aorta and kidney artery, and showed evidence of insulin resistance. These are all major risk factors for heart disease, stroke and kidney failure. The high fructose plus high salt diet also caused early dysfunction of the left ventricle of the heart. Treatment with an antioxidant prevented the hypertension, insulin resistance, and aortic stiffness but did not benefit renal artery changes or poor function of the left ventricle of the heart.

\section{Introduction}

Hypertension prevalence is currently estimated at $46 \%$ among adults in the United States and remains one of the top healthcare and societal burdens. ${ }^{1}$ Chronic increases in arterial pressure contribute to the development of comorbidities and major adverse cardiac events (MACE) such as stroke, myocardial infarction, heart failure, and renal disease. ${ }^{2}$ Despite the urgent need to implement strategies for incidence reduction, the etiologies underlying the majority of hypertensive states still remain unknown. In addition to genomic factors, environmental factors including suboptimal dietary habits also contribute to the development of hypertension. Consumption of fructose has been on the rise due to increasing use of high fructose corn syrup since the 1970s for sweetening beverages and pre-packaged foods. ${ }^{3}$ A recent meta-analysis found that individuals consuming sugarsweetened beverages in the highest versus lowest quantile displayed a greater risk for hypertension. ${ }^{4}$ The association between fructose ingestion and hypertension is potentiated by increased salt intake. ${ }^{5}$ Indeed, a typical Western diet is enriched in both fructose and salt.

Previous work in the fructose-induced salt-sensitive hypertension rat model showed increased reactive oxygen species (ROS) production, diminished nitric oxide bioavailability and cumulative positive sodium balance. ${ }^{6,7}$ Activation of the renin-angiotensin-aldosterone system (RAAS) and increased renal sympathetic nerve activity, both of which promote increased renal tubular sodium reabsorption, have also been implicated as mechanisms resulting in elevated arterial pressure. ${ }^{6,8-11}$ Notably, bilateral renal cryo-denervation ${ }^{9}$ or daily treatment with the superoxide dismutase mimetic, 4-hydroxy-2,2,6,6-tetramethylpiperidine-1-oxyl (+T; Tempol), reduces 8-isoprostane excretion and ameliorates the hypertension. ${ }^{10}$ In light of these findings, it is possible that combined fructose and high salt diet may exert a deleterious impact on cardiac and vascular function either directly or indirectly via the elevated arterial pressure. If so, then it would be important to evaluate whether 4-hydroxy-2,2,6,6-tetramethylpiperidine-1-oxyl also ameliorates cardiovascular dysfunction in this model.

Data from the Framingham population highlight that aortic stiffening precedes hypertension. ${ }^{12,13}$ The force of ejected blood during cardiac systole exerts pressure on the aortic wall that makes the normal aorta expand and then recoil back during cardiac diastole. This property of healthy aorta is known as the Windkessel function, or capacitance, and functionally is responsible for reduction of systolic blood pressure and maintenance of peripheral perfusion during diastole. As the aorta stiffens such as occurs physiologically in aging and pathophysiologically in diabetes, obesity and hypertension, it loses the Windkessel function which is reflected in increases in pulse wave velocity (PWV). ${ }^{14,15}$ Accordingly, aortic PWV is one of the most reliable predictors of cardiovascular mortality as demonstrated by two major metaanalyses. $^{16,17}$ Elastic properties of major vessels can also be assessed with strain imaging as demonstrated by a recent study describing reductions in common carotid artery strain as a valuable tool for detection of atherosclerotic disease. ${ }^{18}$ Likewise, a decrease in ascending aortic strain is an independent predictor of significant coronary artery disease in patients with coronary artery stenosis $\geq 70 \%{ }^{19}$ Given the interdependence of systemic and renal hemodynamics, it is tempting to speculate that aortic stiffening is expected to have deleterious consequences on the renal vasculature. Renal resistive index (RRI) measured with intrarenal Doppler ultrasound is influenced by complex interactions between vascular wall properties and systemic factors and can be used to identify vascular dysfunction. ${ }^{20}$ Higher RRI is associated with increased $\mathrm{PWV}^{21}$ and leads to microvascular damage over time. $^{22}$ The effects of fructose-induced salt-sensitive hypertension on the elastic properties of conduit and resistance vessels are presently undefined. Even in the absence of hypertension, salt-sensitivity in humans constitutes a cardiovascular risk factor. ${ }^{23,24}$ It is unknown whether fructose predisposes to salt-sensitive hypertension by reduced aortic compliance, increased PWV and consequential perturbations in renal hemodynamics or via the ROS system. However, such a process could link fructose and high salt ingestion with hypertension and may explain how fructose-induced salt-sensitive hypertension leads to systemic and renal hemodynamic derangements.

In the present investigation, we employed the fructoseinduced salt-sensitive hypertension rat model by feeding rats 
$20 \%(\mathrm{w} / \mathrm{v})$ fructose for one week, followed by the addition of $4 \% \mathrm{NaCl}$ for three more weeks, which results in increases in mean arterial pressure. ${ }^{9}$ We hypothesized that dietary fructose, rather than glucose, in combination with high salt will lead to aortic stiffening, evidenced by increased aortic PWV, decreased strain, and decreased distensibility coefficient. This will, in turn, result in an increase in RRI. We hypothesize that daily supplementation with the antioxidant, 4-hydroxy2,2,6,6-tetramethylpiperidine-1-oxyl, will ameliorate the increase in mean arterial pressure, and thereby mitigate vascular stiffening. These studies are aimed at identifying the diverse mechanisms that diminish aortic compliance, impair renal vascular function, and contribute to hypertension.

\section{Materials and Methods Animals}

Male Sprague Dawley rats (Harlan Sprague Dawley, Indianapolis, IN) were housed under controlled conditions $\left(21-23^{\circ} \mathrm{C} ; 12 \mathrm{hr}\right.$ light and $12 \mathrm{hr}$ dark cycles, light on at 7 am) and were permitted ad libitum access to water and standard rat chow containing $0.4 \% \mathrm{NaCl}$ until they were enrolled into experimental protocols described below. Complete care provided to rats was in accordance with the principles of the National Institutes of Health Guide for the Care and Use of Laboratory Animals. All procedures and protocols were approved by the Wayne State University Institutional Animal Care and Use Committee (Protocol \#19-01-1001).

\section{Dietary Regimen}

Upon arrival of animals to the DLAR facilities, all rats were provided ad libitum access to $0.4 \% \mathrm{NaCl}$ standard chow (Envigo Teklad, Madison, WI) and normal water. Three to five days later, hemodynamic telemetry devices were implanted and rats allowed to recover for three-four days in single housing. Following previously established protocols, ${ }^{6,9,10}$ the rats were randomized to either $20 \%(\mathrm{w} /$ v) glucose (Sigma-Aldrich, St. Louis, MO) or $20 \%(\mathrm{w} / \mathrm{v})$ fructose (Sigma-Aldrich, St. Louis, MO) and maintained $0.4 \% \mathrm{NaCl}$ chow. The age of rats at enrollment was $10-12$ weeks and the average weight was $228.6 \pm 5.9 \mathrm{~g}$ in the control group and $225 \pm 2.5 \mathrm{~g}$ in the Tempol group. Glucose was provided in the control groups to match both caloric and fluid intake. The consumption of glucose and fructose was similar throughout the protocol ( 65 calories per day as carbohydrate). After one week, the rats were randomly assigned to one of eight groups: 1) glucose and normal (0.4\%) salt (Glu+NS), 2), glucose and high $(4.0 \%)$ salt (Glu+HS), 3) fructose and normal salt $($ Fru+NS), 4) fructose and high salt (Fru+HS), 5) Glu+NS with the addition of 4-hydroxy-2,2,6,6-tetramethylpiperidine-1-oxyl (+T) (Glu+NS+T), 6) Glu+HS+T, 7) Fru-NS+T or 8) Fru+HS+T.

To assure that each rat in the groups receiving $+\mathrm{T}$ (Santa Cruz, Santa Cruz, CA) received the full dose, $35 \mathrm{~mL}$ of the respective drinking mixture was supplemented with $+\mathrm{T}, 15 \mathrm{mg} / 400 \mathrm{~g}$ body weight. After the rat completed drinking the allotted antioxidant solution, a fresh water bottle with the respective glucose or fructose supplement alone was provided. This approach allowed verification that each rat received its full dose of antioxidant and that fructose or glucose ingestion across all groups was similar. The respective dietary regimens were continued for three weeks with monitoring of hemodynamic parameters via telemetry.

\section{Surgical Procedures} Hemodynamic Telemetry Transmitter Placement

All rats were instrumented with hemodynamic telemeters at ten weeks of age ( $225 \mathrm{~g}$ body weight) under ketamine $(80 \mathrm{mg} / \mathrm{kg})$ and xylazine $(10 \mathrm{mg} / \mathrm{mL})$ anesthesia. An incision was made in the groin and a hemodynamic telemetry transmitter (TA11PA-C40, Data Sciences International) implanted by exposing the right femoral artery, temporarily occluding the proximal end, and inserting the gel-filled catheter attached to the transmitter device into the artery with a 21-gauge needle. The catheter was advanced into the abdominal aorta and the transmitter tunneled subcutaneously. The incision was closed using surgical staples. Post-operative analgesia was provided with buprenorphine SR (0.3 mg/kg subcutaneously).

\section{Vascular Catheter Placement}

At the end of the fourth week (1-week glucose/fructose +3 weeks \pm Tempol $^{\circledR}$ ), the rats were instrumented with carotid arterial catheters for hemodynamic monitoring during ultrasonography and for collection of aortic blood at the conclusion of experiments.

\section{Telemetry, Echocardiography and Renal Doppler Studies}

Data acquisition of hemodynamic parameters was performed using Dataquest ART software (Data Sciences International). Measurements of heart rate, systolic (SBP), diastolic (DBP), and mean arterial pressures (MAP) as well as the derived 
pulse pressure (PP) were sampled for $10 \mathrm{sec}$ at a sampling rate of 500 samples/sec every $1 \mathrm{hr}$ for the remainder of the 3 -week protocol. The average of hemodynamic recordings initiated during the last three days of the week during which the rats were on glucose or fructose and $0.4 \% \mathrm{NaCl}$ were taken as baseline. Once assigned to one of the eight groups (see above), hemodynamic measurements over the next three weeks were taken for $24 \mathrm{hr}$ beginning at 7 am (lights on) during Mondays and Thursdays in order to avoid cage cleaning days and disruptions (eg, weekend changes in veterinary staff) that could impact the parameters as well as conserve telemeter battery time. Values are the 24-hr average for a given day. Two days after placement of vascular catheters, echocardiography and renal Doppler scans were completed by an investigator blinded to the group assignments. Blood pressure and heart rate were monitored continuously with PowerLab (ADInstruments, Colorado Springs, CO) connected to the LabChart 8.0 software.

Rats were anesthetized with 3\% isoflurane in an induction chamber, and maintenance of anesthesia was achieved with $1.5 \%$ isoflurane delivered via a nose cone. Scans were performed on the heated platform (Fujifilm Visualsonics, Inc., Toronto, Canada) in a supine position with all four limbs secured to ECG electrodes with tape. Fur was removed from the chest and abdominal areas first by shaving the area followed by a cream depilatory (Pharmaceutical Innovations, Newark, NJ), before applying contact gel preheated to $37^{\circ} \mathrm{C}$. Body temperature was monitored using a rectal probe. Echocardiographic recordings were made according to standard methods ${ }^{25,26}$ at the conclusion of the three-week dietary intervention.

Images were acquired using a Vevo3100 with an MX$250 \mathrm{~S}$ transducer (Fujifilm Visualsonics Inc.). A parasternal short axis view was used to obtain images in M-mode at the level of papillary muscles to assess systolic function and left ventricular dimensions. Left ventricular diastolic function was ascertained using pulse wave Doppler recordings of transmitral flow velocities aligned in the apical four chamber view. Aortic PWV was determined using B-mode and pulse wave Doppler of aortic flow velocities at two positions along the aortic arch. ${ }^{27}$ Briefly, the time to initiation of flow was determined relative to the ECG and the difference calculated. The distance between the two flow positions determined along the B-mode image, was then divided by the time to calculate the PWV.

The renal resistive index (RRI) was ascertained using pulse wave Doppler imaging of flow through the left main renal artery. The RRI was calculated as the difference between the peak systolic and minimum diastolic velocity, divided by the peak systolic velocity. ${ }^{20}$ Aortic distensibility and strain were derived from the B-mode images. Some measurements were not able to be technically ascertained in each animal due to anatomical constraints. Data analysis was completed offline in blinded fashion using VevoLab and VevoVasc software (Fujifilm Visualsonics, Inc.).

After all scans were completed, the rats were permitted to recover from isoflurane anesthesia and allowed to regain normal activities for $\sim 4-6 \mathrm{hr}$. Arterial blood was then collected in the conscious animals using the carotid catheter for hormone analyses. Blood $(1 \mathrm{~mL})$ was taken over 90 -sec period into pre-chilled tubes containing $50 \mu \mathrm{L}$ sodium ethylenediaminetetraacetic acid (EDTA) for PRA. Another $1.5 \mathrm{~mL}$ of blood was taken into a separate prechilled tube with $120 \mu \mathrm{L}$ of $500 \mathrm{mM}$ sodium EDTA, 125 $\mathrm{mM}$ phenanthroline, $1 \mathrm{mM}$ phenylmethanesulfonyl fluoride, $20 \mathrm{mM}$ pepstatin, $1 \mathrm{mM}$ enalapril and 10X phosphatase inhibitor cocktail for Ang II. The blood was immediately centrifuges at $3000 \mathrm{rpm}$ at $4^{\circ} \mathrm{C}$, and the plasma stored at $-70^{\circ} \mathrm{C}$ until assay.

The rats were then euthanized with pentobarbital $(100 \mathrm{mg} / \mathrm{kg})$ and hearts and kidneys harvested and weighed while keeping them cold in saline. The renal cortex was then rapidly and sharply dissected and the tissue frozen at $-80^{\circ} \mathrm{C}$ until analyzed for tissue Ang II.

\section{Hormone Assays}

All tests were performed on blood samples obtained simultaneously between $1 \mathrm{pm}$ and $4 \mathrm{pm}$. Non-fasting blood glucose concentrations were measured with One Touch Ultra 2 (Lifescan, Switzerland). Plasma renin activity (PRA) (IBL International, Hamburg, Germany) and insulin (Cayman, Ann Arbor, MI) were measured by enzymelinked immunosorbent assays according to the product directions. Plasma and tissue angiotensin II (Ang II) concentrations were assessed by radioimmunoassay as previously described by our laboratory. ${ }^{28}$

\section{Analyses and Statistics}

Heart rate and arterial pressures were collected using Dataquest ART software (Data Sciences Intl, St. Paul, MN) and averaged over a 24-hr period. All analyses were performed by a researcher blinded to the group assignments. Results are presented as mean $\pm \mathrm{SE}$. Comparisons among groups were completed with one-way ANOVA; comparisons among groups over time were accomplished using two-factor ANOVA. A repeated measures design requires that every 
participant participates in every condition of the experiment, historically called a within-subjects design. Our data were unbalanced, that is, there were slightly different but unequal sample sizes across each group since some procedures were not able to be performed in each animal due to technical reasons (eg, catheter malfunction). The repeated measures design drops sample sizes in the analysis until the same, or equal, numbers are in each group across all time points. Ideally, the repeated measures design (with time point) would be preferred. However, this selection of analysis would drop the already small sample size until the numbers were equal across all time points and in each group observed until they all have the same sample size. The two-factor approach (group and time point) allows us to keep all the data available and conduct mean comparisons, using a Sidak post hoc test for multiple pairwise comparisons. Homogeneity of variance was checked and verified using Leven's test.

Comparisons of ultrasound parameters between Fru $+\mathrm{HS}$ and Fru+HS+T groups were done using Student's unpaired $t$-test. Differences were deemed statistically significant when the P-value was less than 0.05 .

\section{Results}

\section{Systemic Hemodynamics, Body and Organ Weights}

Baseline body weight and heart weight of fructose-fed and glucose-fed rats with and without antioxidant supplementation were similar. Kidney weights were significantly greater in control fructose-fed rats on either normal or high salt diets.
In + T-treated rats, mean kidney weights were also higher but the differences were only significant for the left kidneys since right kidney weights displayed greater variability (Table 1).

Baseline SBP, DBP, MAP and heart rate were similar across all groups (Table 2). Baseline PP appeared to be greater in the Fru+HS group but this did not achieve significance $(\mathrm{P}=0.16)$. SBP and MAP increased significantly after three weeks of high salt diet in fructose-fed but not glucose-fed animals; supplementation with antioxidant prevented this increase (Figure 1). After three weeks, the change in DBP, PP and heart rate did not differ among the groups. For clarity, Figure 2 depicts the progression of MAP over time only for fructose-fed rats. By day 10, MAP rose significantly compared with baseline in Fru+HS rats and remained elevated from Day 17 through the 24th day. MAP progressively declined from Day 7 to Day 24 in the Fru+HS+T group such that there was no difference in MAP among Fru+NS, Fru+NS+T and Fru+HS+T groups by day 24; however, MAP of the Fru+HS group was significantly greater than any of the other three groups. SBP followed the same pattern as MAP. Glucose-fed rats in any of the groups did not display significant changes from baseline or among the groups in MAP at any point of time (data not shown).

\section{Cardiovascular Parameters Measured by Echocardiography}

The increase in MAP in the Fru+HS group was accompanied by an increase in aortic pulse wave velocity (PWV)

Table I Rat, Kidney and Heart Weights for All Rat Groups

\begin{tabular}{|c|c|c|c|c|c|c|}
\hline & \multirow[t]{3}{*}{$\mathbf{n}$} & \multirow{3}{*}{$\begin{array}{c}\text { Initial Rat Body Weight } \\
\text { (g) }\end{array}$} & \multirow{3}{*}{$\begin{array}{c}\text { Final Rat Body Weight } \\
\text { (g) }\end{array}$} & \multicolumn{2}{|c|}{ Kidney Weight } & \multirow{3}{*}{$\begin{array}{l}\text { Heart Weight } \\
(\mathrm{g} / \mathrm{kg} \mathrm{BW})\end{array}$} \\
\hline & & & & Left & Right & \\
\hline & & & & \multicolumn{2}{|c|}{ (g/kg BW) } & \\
\hline \multicolumn{7}{|c|}{ Control } \\
\hline Glu+NS & 8 & $230 \pm 8$ & $327 \pm 7$ & $2.9 \pm 0.1$ & $2.9 \pm 0.2$ & $3.8 \pm 0.8$ \\
\hline Glu+HS & 12 & $224 \pm 5$ & $329 \pm 8$ & $2.9 \pm 0.1$ & $2.9 \pm 0.3$ & $3.9 \pm 0.5$ \\
\hline Fru+NS & 8 & $227 \pm 7$ & $330 \pm 13$ & $3.1 \pm 0.1 *$ & $3.2 \pm 0.3^{*}$ & $4.1 \pm 0.7$ \\
\hline Fru+HS & 10 & $226 \pm 5$ & $313 \pm 5$ & $3.2 \pm 0.1^{*}$ & $3.1 \pm 0.3^{*}$ & $3.9 \pm 0.4$ \\
\hline \multicolumn{7}{|c|}{ Tempol } \\
\hline Glu+NS+T & 10 & $222 \pm 5$ & $332 \pm 10$ & $2.9 \pm 0.1$ & $2.9 \pm 0.2$ & $3.7 \pm 0.4$ \\
\hline $\mathrm{Glu}+\mathrm{HS}+\mathrm{T}$ & 12 & $228 \pm 8$ & $329 \pm 5$ & $2.8 \pm 0.1$ & $2.9 \pm 0.3$ & $3.9 \pm 0.4$ \\
\hline Fru+NS+T & 9 & $224 \pm 6$ & $329 \pm 9$ & $3.2 \pm 0.1^{\dagger}$ & $3.2 \pm 0.2$ & $3.7 \pm 0.4$ \\
\hline $\mathrm{Fru}+\mathrm{HS}+\mathrm{T}$ & 9 & $222 \pm 5$ & $337 \pm 14$ & $3.1 \pm 0.1^{\dagger}$ & $3.1 \pm 0.4$ & $3.7 \pm 0.4$ \\
\hline
\end{tabular}

Notes: Values are mean \pm SE. $* P<0.05$ vs corresponding glucose-fed group ${ }^{\dagger} P<0.05$ vs corresponding glucose-fed plus Tempol ${ }^{\circledR}$ group.

Abbreviations: Glu+NS, $20 \%$ glucose $+0.4 \% \mathrm{NaCl}$; Glu+HS, $20 \%$ glucose $+4 \% \mathrm{NaCl}$; Fru+NS, $20 \%$ fructose $+0.4 \% \mathrm{NaCl}$; Fru+HS, $20 \%$ fructose $+4 \% \mathrm{NaCl} ;+\mathrm{T}$, indicates addition of $15 \mathrm{mg} / 400 \mathrm{~g} \mathrm{BW}$ Tempol ${ }^{\circledR}$; BW, body weight. 
Table 2 Baseline Hemodynamics and Changes After 3-Week Diet Regimen

\begin{tabular}{|c|c|c|c|c|c|c|c|c|c|c|c|}
\hline Diet & $\mathbf{N}$ & $\begin{array}{l}\text { Baseline } \\
\text { MAP } \\
(\mathrm{mmHg})\end{array}$ & $\begin{array}{c}\Delta M A P \\
(\mathbf{m m H g})\end{array}$ & $\begin{array}{l}\text { Baseline } \\
\text { SBP } \\
(\mathbf{m m H g})\end{array}$ & $\begin{array}{c}\Delta S B P \\
(\mathbf{m m H g})\end{array}$ & $\begin{array}{l}\text { Baseline } \\
\text { DBP } \\
(\mathrm{mmHg})\end{array}$ & $\begin{array}{c}\Delta D B P \\
(\mathrm{mmHg})\end{array}$ & $\begin{array}{c}\text { Baseline } \\
\text { PP } \\
(\mathrm{mmHg})\end{array}$ & $\begin{array}{c}\Delta P P \\
(\mathbf{m m H g})\end{array}$ & $\begin{array}{c}\text { Baseline } \\
\text { Heart Rate } \\
\text { (bpm) }\end{array}$ & $\begin{array}{c}\Delta \mathrm{HR} \\
\text { (bpm) }\end{array}$ \\
\hline \multicolumn{12}{|c|}{ Control } \\
\hline Glu+NS & 8 & $107 \pm 3$ & $0.2 \pm 1.5$ & $123 \pm 4$ & $0.3 \pm 1.7$ & $93 \pm 5$ & $4.7 \pm 1.5$ & $29 \pm 3$ & $1.6 \pm 2.2$ & $4 I I \pm 20$ & $-28 \pm 20$ \\
\hline Glu+HS & 12 & $106 \pm 2$ & $1.5 \pm 1.0$ & $122 \pm 2$ & $1.9 \pm 1.2$ & $93 \pm 3$ & $5.6 \pm 2.8$ & $30 \pm 2$ & $-1.6 \pm 1.6$ & $437 \pm 27$ & $-30 \pm 20$ \\
\hline Fru+NS & 8 & $107 \pm 3$ & $-0.9 \pm 1.8$ & $124 \pm 4$ & $-1.3 \pm 2.5$ & $93 \pm 4$ & $5.6 \pm 2.1$ & $31 \pm 3$ & $1.2 \pm 2.6$ & $408 \pm 21$ & $-26 \pm 26$ \\
\hline Fru+HS & 10 & $107 \pm 2$ & $5.5 \pm 0.8^{* \ddagger}$ & $126 \pm 4$ & $5.6 \pm 1.5^{* \neq}$ & $91 \pm 2$ & $6.7 \pm 2.8$ & $37 \pm 3$ & $0.9 \pm 1.7$ & $409 \pm 19$ & $-16 \pm 20$ \\
\hline \multicolumn{12}{|c|}{ Tempol } \\
\hline Glu+NS+T & 10 & $104 \pm 2$ & $1.2 \pm 1.1$ & $|2| \pm 4$ & $-0.4 \pm 2.0$ & $93 \pm 3$ & $4.9 \pm 1.8$ & $28 \pm 3$ & $-0.3 \pm 2.0$ & $431 \pm 24$ & $-25 \pm 11$ \\
\hline $\mathrm{Glu}+\mathrm{HS}+\mathrm{T}$ & 12 & $107 \pm 2$ & $1.1 \pm 1.1$ & $|2| \pm 4$ & $2.7 \pm 1.2$ & $87 \pm 3$ & $5.1 \pm 3.5$ & $34 \pm 3$ & $-0.8 \pm 1.2$ & $421 \pm 22$ & $-26 \pm 20$ \\
\hline $\mathrm{Fru}+\mathrm{NS}+\mathrm{T}$ & 9 & $108 \pm 3$ & $1.2 \pm 0.8$ & $128 \pm 4$ & $0.3 \pm 1.1$ & $98 \pm 6$ & $6.1 \pm 3.0$ & $29 \pm 4$ & $-0.6 \pm 0.7$ & $422 \pm 15$ & $-31 \pm 13$ \\
\hline Fru+HS+T & 9 & $110 \pm 2$ & $0.8 \pm 0.8^{\dagger}$ & $129 \pm 3$ & $1.9 \pm 0.7^{\dagger}$ & $91 \pm 1$ & $5.3 \pm 3.3$ & $39 \pm 3$ & 0.91 .5 & $422 \pm 13$ & $-23 \pm 16$ \\
\hline
\end{tabular}

Notes: Group definitions as in Table I. Values are mean \pm SE. $* P<0.05$ vs Glu+NS; ${ }^{\ddagger} P<0.05$ vs Fru+NS; ${ }^{\dagger} P<0.05$ vs Fru+HS.

$(803 \pm 19 \mathrm{~mm} / \mathrm{s}, P<0.005)$ compared with Glu+NS $(606 \pm$ $48 \mathrm{~mm} / \mathrm{s})$, Glu+HS $(645 \pm 39 \mathrm{~mm} / \mathrm{s})$ or Fru+NS $(700 \pm$ $27 \mathrm{~mm} / \mathrm{s}$ ) groups (Figure 2A). Attenuation of free radical formation decreased aortic PWV in Fru+HS+T rats $(686 \pm$ $31 \mathrm{~mm} / \mathrm{s}, P<0.005)$ compared to their non-treated counterparts (Figure 2B), while it produced no change in PWV in other groups (Glu+NS: $681 \pm 30 \mathrm{~mm} / \mathrm{s}$, Glu+HS+T: $630 \pm$ $37 \mathrm{~mm} / \mathrm{s}$ and Fru+NS+T: $684 \pm 43 \mathrm{~mm} / \mathrm{s}$ ).

Echocardiographic measures of systolic cardiac function revealed no overt phenotype in rats fed fructose and high salt diet. Ejection fraction, fractional shortening, left ventricle (LV) dimensions, and LV mass were similar across all groups (Table 3). Notably, only the Fru+HS rats displayed evidence of diastolic dysfunction (Figure 3A). Specifically, the ratio of early (E) to late (A) transmitral filling velocities was decreased in Fru+HS rats $(1.12 \pm 0.03$,

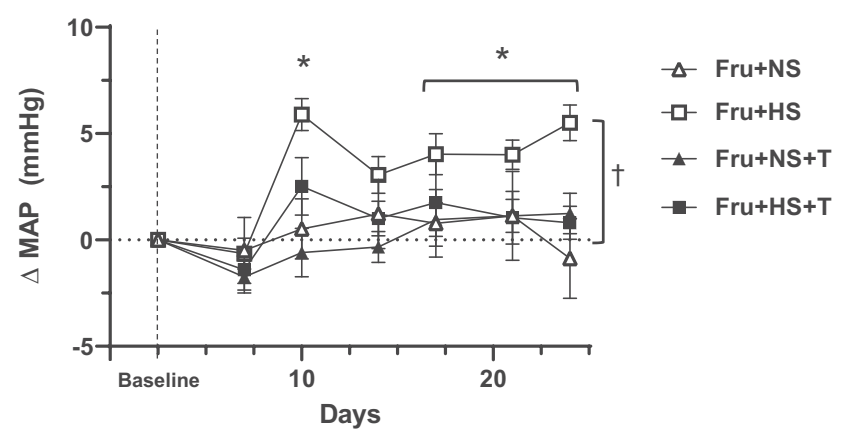

Figure I Change in mean arterial pressure (MAP) over the 3-week period for the fructose-fed groups. Baseline MAP and delta MAP for glucose-fed groups did not change significantly over time (Table 2 ); not depicted graphically for simplicity. Fru $+\mathrm{NS}, 20 \%$ fructose $+0.4 \% \mathrm{NaCl}$; Fru+HS, $20 \%$ fructose $+4 \% \mathrm{NaCl}$; $+\mathrm{T}$, indicates addition of $15 \mathrm{mg} / 400 \mathrm{~g} \mathrm{BW}$ Tempol; BW, body weight. *Values are mean $\pm \mathrm{SE}$; $\mathrm{n}$ as in Table $2 . * P<0.05$ vs baseline for Fru+HS; ${ }^{\dagger} P<0.05$ for Fru+HS vs all other fructose-fed groups by two-way ANOVA on Day 24.
$P<0.005)$ compared with the rats on glucose (Glu+NS: $1.28 \pm 0.04$ and Glu+HS: $1.37 \pm 0.05)$ or Fru+NS rats $(1.37 \pm 0.08)$. Supplementation with the antioxidant did not change E/A in Fru+HS rats $(1.09 \pm 0.07$, Figure 3B) nor in any of the other groups: Glu+NS+T $(1.38 \pm 0.07)$, $\mathrm{Glu}+\mathrm{HS}+\mathrm{T}(1.25 \pm 0.06)$ and Fru+NS+T (1.28 \pm 0.04$)$.

Radial ascending aortic strain was decreased in rats fed fructose and high salt $(11.60 \pm 0.76 \%, P<0.05)$ compared with Glu+NS (16.24 $\pm 1.33 \%)$, Glu+HS $(18.90 \pm 2.44 \%)$ or Fru+NS $(17.39 \pm 1.93 \%$ ) groups (Figure 4A). This was also reflected in reductions in the distensibility coefficient in Fru $+\mathrm{HS}$ rats $\left(44.22 \pm 7.70 \mathrm{MPa}^{-1}, \mathrm{P}<0.05\right)$, compared with Glu+NS $\left(88.37 \pm 8.02 \mathrm{MPa}^{-1}\right)$, Glu+HS $(80.30 \pm 10.15$ $\left.\mathrm{MPa}^{-1}\right)$ or Fru+NS $\left(92.49 \pm 23.88 \mathrm{MPa}^{-1}\right)$ groups (Figure 4C). Treatment with superoxide dismutase mimetic reversed the impairment of the aorta in Fru+HS rats to expand and accommodate the ejected blood (Fru $+\mathrm{HS}+\mathrm{T}$, $16.25 \pm 1.38 \%, P<0.05)$, thereby increasing radial ascending aortic strain (Figure 4B). Radial ascending aortic strain remained unchanged in $+\mathrm{T}$-treated groups on all other diets (Glu+NS, $17.68 \pm 2.61 \%$; Glu+HS, $18.67 \pm 3.16 \%$; and Fru $+\mathrm{NS}, 17.62 \pm 2.74 \%$ ). On the other hand, Figure 4D shows that treatment with $+\mathrm{T}$ increased the distensibility coefficient in fructose-fed rats on high salt diet (Fru+HS+T, 64.59 $\pm 10.48 \mathrm{MPa}^{-1}$ ) but did not restore it to levels comparable to those in the glucose-fed groups.

The RRI, a measure of renal vascular stiffness or impedance, was significantly augmented in the Fru+HS group $(0.60 \pm 0.03, P<0.05)$, compared with Glu+NS $(0.45 \pm$ $0.03)$, Glu + HS $(0.45 \pm 0.04)$ or Fru+NS groups $(0.47 \pm 0.05)$ (Figure 5A). No differences were observed among the +T-treated groups $(\mathrm{Glu}+\mathrm{NS}+\mathrm{T}, 0.50 \pm 0.04$; Glu+HS+T, 
A

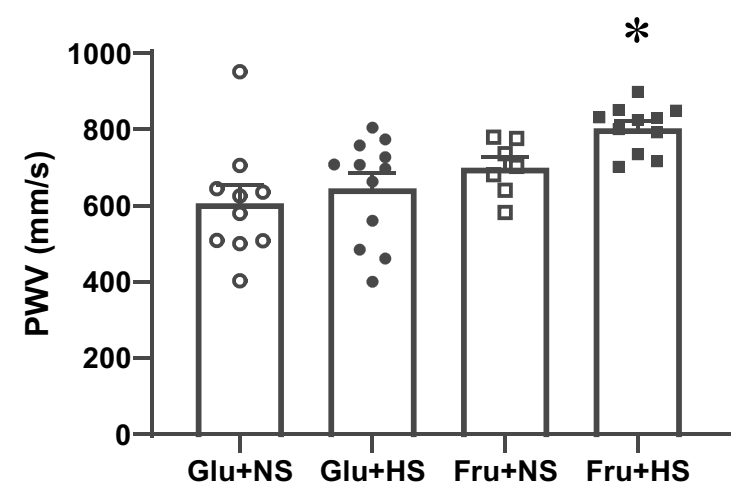

B

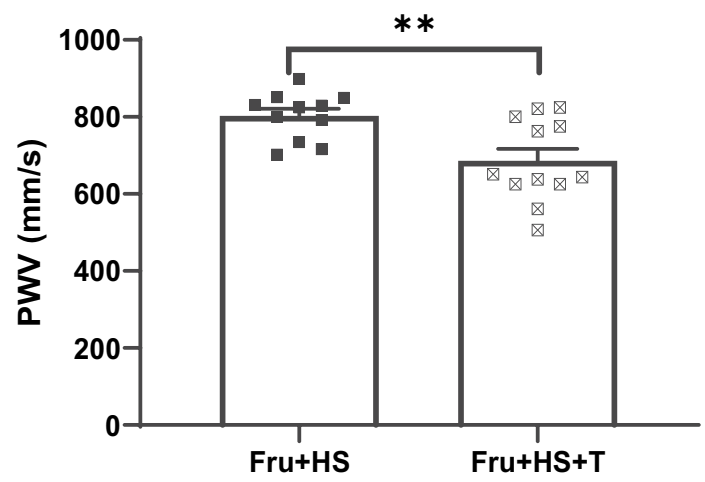

Figure 2 Aortic pulse wave velocity (PWV) in (A) the four groups of rats: Glu+NS, $20 \%$ glucose $+0.4 \% \mathrm{NaCl} ; \mathrm{Glu}+\mathrm{HS}, 20 \%$ glucose $+4 \% \mathrm{NaCl}$; Fru+NS, $20 \%$ fructose $+0.4 \%$ $\mathrm{NaCl}$; Fru+HS, $20 \%$ fructose $+4 \% \mathrm{NaCl}$. (B) Aortic pulse wave velocity in rats given either Fru+HS alone or with addition of $15 \mathrm{mg} / 400 \mathrm{~g} \mathrm{BW}$ Tempol (+T); $\mathrm{BW}$, body weight, Fru+HS+T. Values are mean $\pm \mathrm{SE} ; \mathrm{n}$ as indicated per group; $* P<0.005$ vs other three groups; $* * P<0.005$ vs Fru+HS group.

$0.53 \pm 0.4 ;$ Fru $+\mathrm{NS}+\mathrm{T}, 0.50 \pm 0.02$; and Fru+HS+T, $0.60 \pm$ $0.05)$. Notably, the antioxidant failed to reverse the augmented RRI in rats fed fructose and high salt diet (Figure 5B).

\section{PRA, Angiotensin II and Insulin Levels}

High salt diet did not suppress PRA in either glucose-fed or fructose-fed rats (Table 4). In contrast, plasma Ang II was significantly lower in both Glu+HS and Fru+HS rats compared with rats fed the same sugar on normal salt diet. Plasma Ang II was attenuated in both Glu+NS+T and Fru+NS+T groups such that there were no differences among any of the +T-treated groups. Although kidney cortical tissue Ang II tended to be higher in the + T-treated normal salt groups vs the $+\mathrm{T}$-treated high salt groups ingesting the same sugar, renal cortical tissue Ang II levels did not significantly differ among any of the groups. Non-fasting blood glucose values were similar among the groups (Table 4). Figure 6A shows that the glucose:insulin ratio was significantly diminished in the Fru + HS group $(\mathrm{P}<0.05)$, indicative of insulin resistance compared with the other groups. The glucose:insulin ratio was restored in the Fru+HS+T group to a value significantly greater than the Fru+HS group (Figure 6B, P $<0.05$ ).

\section{Discussion}

The major findings of the present investigation support the hypothesis that fructose and high salt feeding for as short a period as three weeks in rats results in salt-sensitive systolic hypertension, stiffening of the ascending aorta, and diastolic left ventricular dysfunction. This occurs at a time when the rats have insulin resistance but are not frankly diabetic. Free radical scavenging at least partially reverses the aortic stiffening but not the diastolic dysfunction. The vascular perturbations associated with combined fructose and high salt intake include increased ascending

Table 3 Echocardiographic Parameters in Control Groups

\begin{tabular}{|l|c|c|c|c|}
\hline & Glu+NS & Glu+HS & Fru+NS & Fru+HS \\
\hline N & 8 & 12 & 7 & 11 \\
LVEF (\%) & $82 \pm 3$ & $78 \pm 3$ & $85 \pm 2$ & $75 \pm 3$ \\
LVFS (\%) & $52 \pm 3$ & $48 \pm 3$ & $56 \pm 3$ & $47 \pm 3$ \\
LVAWs (mm) & $3.11 \pm 0.15$ & $2.92 \pm 0.12$ & $3.31 \pm 0.10$ & $2.82 \pm 0.09$ \\
LVAWd (mm) & $1.95 \pm 0.06$ & $1.98 \pm 0.05$ & $2.09 \pm 0.09$ & $1.85 \pm 0.04$ \\
LVIDs (mm) & $3.07 \pm 0.32$ & $3.22 \pm 0.22$ & $2.72 \pm 0.32$ & $2.58 \pm 0.21$ \\
LVIDd (mm) & $6.34 \pm 0.19$ & $6.34 \pm 0.17$ & $6.34 \pm 0.27$ & $6.58 \pm 0.19$ \\
LVPWs (mm) & $2.94 \pm 0.18$ & $3.17 \pm 0.16$ & $2.92 \pm 0.15$ & $2.81 \pm 0.19$ \\
LVPWd (mm) & $1.84 \pm 0.09$ & $1.96 \pm 0.13$ & $1.64 \pm 0.14$ & $1.84 \pm 0.22$ \\
LV mass (mg) & $645 \pm 39$ & $688 \pm 38$ & $663 \pm 64$ & $631 \pm 33$ \\
\hline
\end{tabular}

Notes: Group definitions as in Table I. Values are mean \pm SE.

Abbreviations: LVEF, left ventricular ejection fraction; LVFS, left ventricular fractional shortening; HR, heart rate; LVAWs, left ventricular end-systolic wall thickness; LVAWd, left ventricular end-diastolic anterior all thickness; LVIDs, left ventricular end-systolic inner diameter; LVIDd, left ventricular end-diastolic inner diameter; LVPWs, end-systolic left ventricular posterior wall thickness; LVPWd, end-diastolic left ventricular posterior wall thickness. 
A

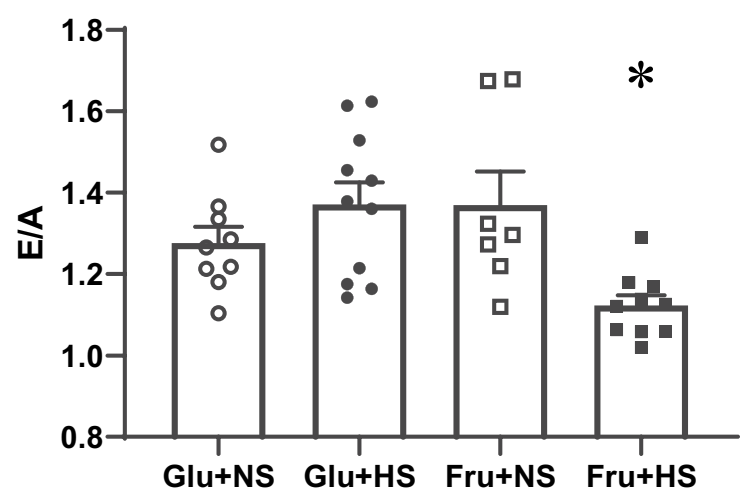

B

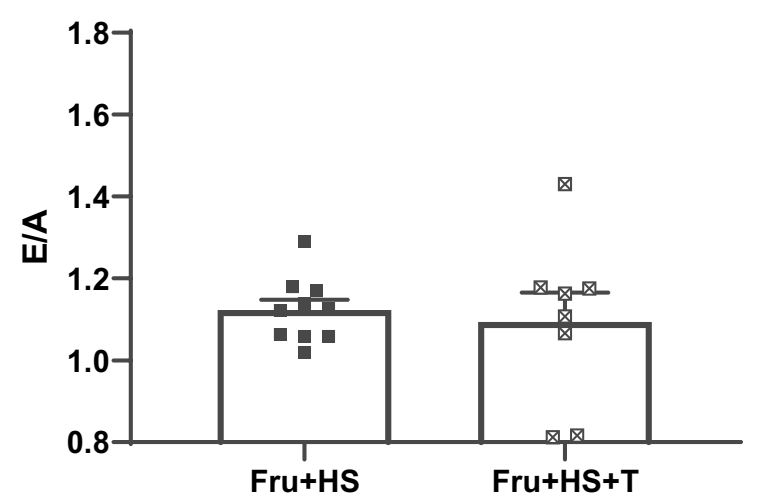

Figure 3 Ratio of early to late (E/A) transmitral filling velocities in $(\mathbf{A})$ the four groups of rats and (B) rats fed fructose-high salt alone or with Tempol (+T). Group designations as in Figure 2. Values are mean $\pm \mathrm{SE} ; \mathrm{n}$ as indicated per group; $* P<0.005$ vs other three groups.
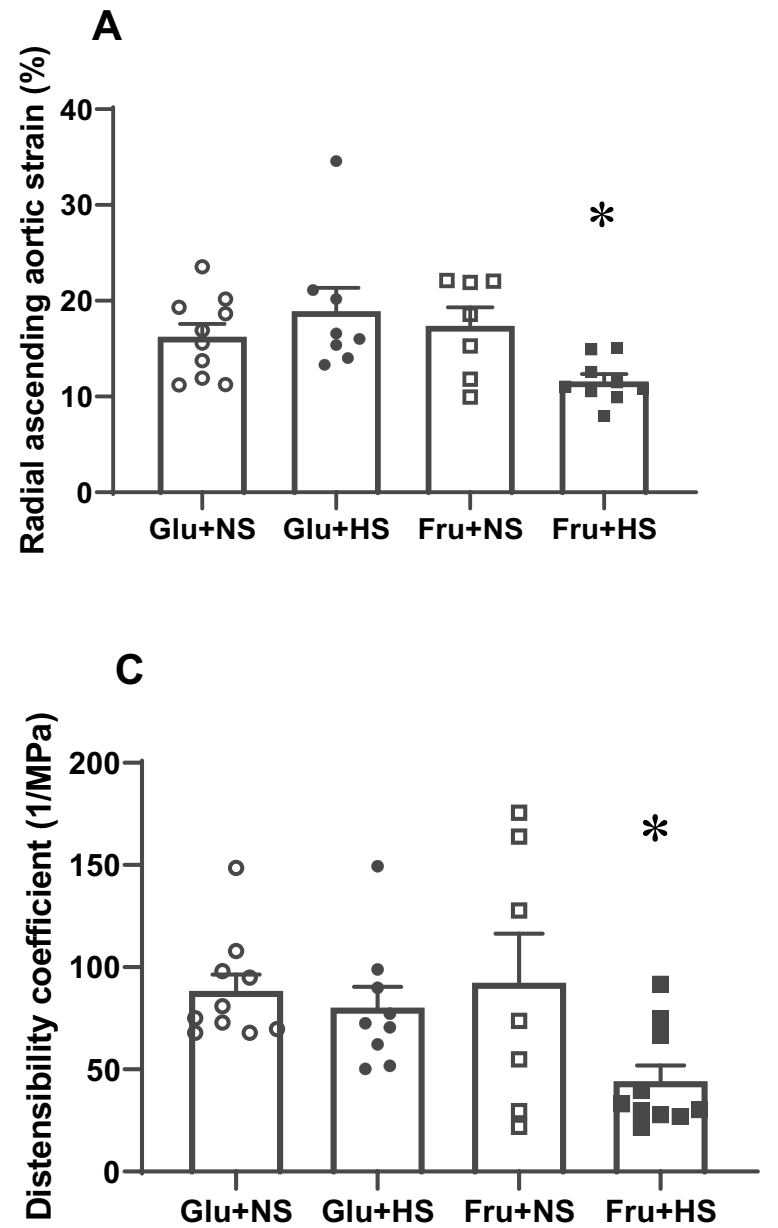
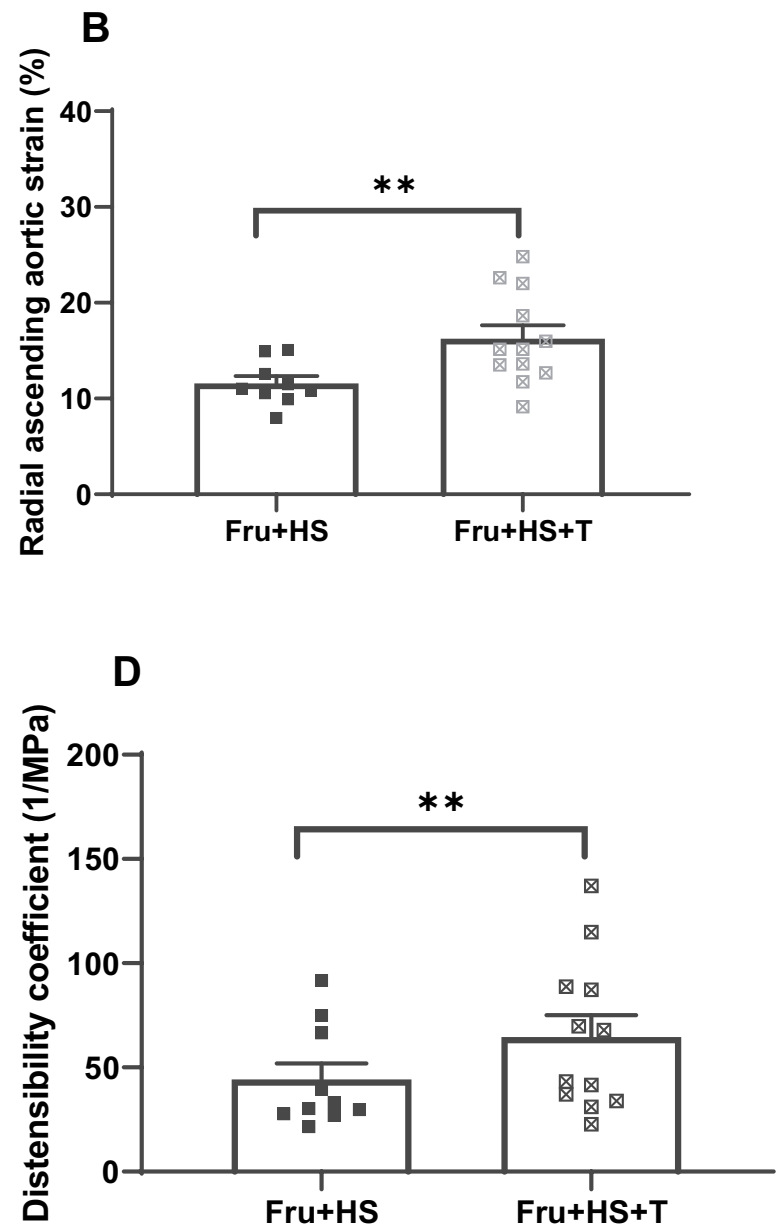

Figure 4 Elastic properties of the ascending aorta: radial ascending aortic strain and distensibility coefficient, respectively, in the four groups of rats (A and $\mathbf{C})$ and in rats fed fructose-high salt alone or with Tempol (B and $\mathbf{D})$. Group designations as in Figure 2 . Values are mean $\pm \mathrm{SE}$; $\mathrm{n}$ as indicated per group; $* \mathrm{P}<0.05$ vs other control groups; $* * \mathrm{P}<$ 0.05 vs Fru+HS group. 

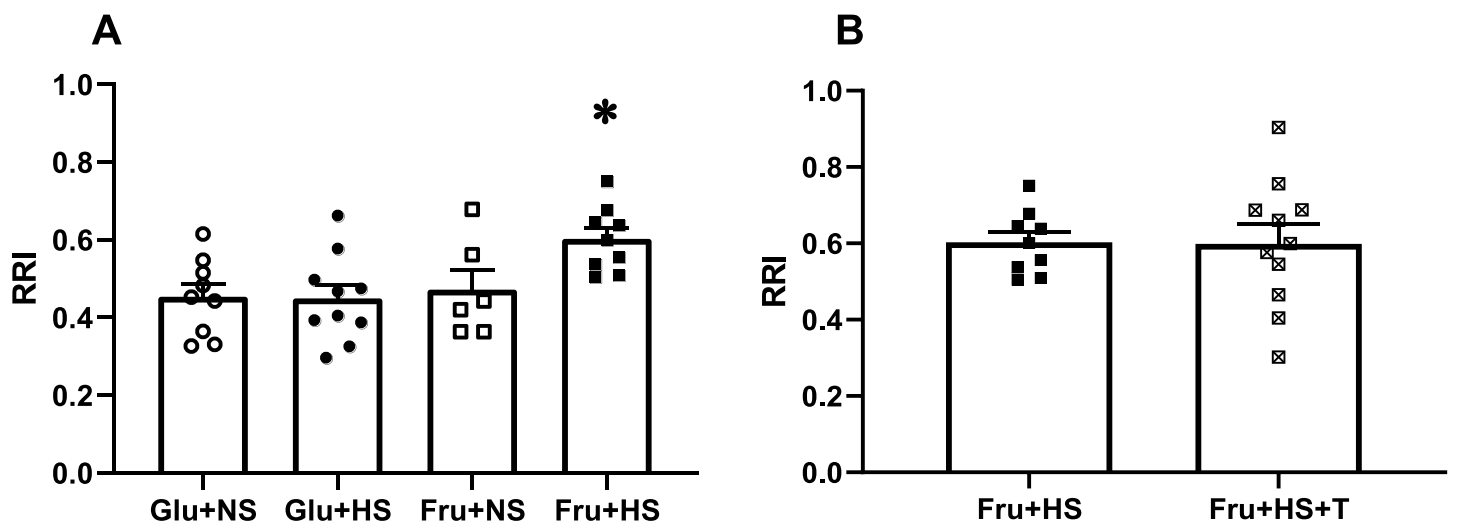

Figure 5 Renal resistive index (RRI) in (A) the four groups of rats and (B) rats fed fructose-high salt alone or with Tempol (+T). Group designations as in Figure 2. Values are mean \pm SE; $n$ as indicated per group; $* P<0.05$ vs other control groups.

aortic PWV, decreased radial ascending aortic strain, and an impaired distensibility coefficient. The decreased E/A ratio denotes early diastolic left ventricular dysfunction. In addition, the increase in RRI in fructose-fed, high salt rats indicates impaired vascular compliance of the main renal artery that is not ameliorated by 4-hydroxy-2,2,6,6- tetramethylpiperidine-1-oxyl, suggesting a mechanistic basis other than oxidative stress. Interestingly, although PRA is not suppressed, the vasoactive hormone plasma Ang II is reduced after three weeks of high salt diet in both glucoseand fructose-fed animals. Intrarenal Ang II does not appear to be affected by either diet or antioxidant treatment.

\section{Fructose, Salt and Blood Pressure}

The present studies confirm previous findings from our own ${ }^{9}$ and other laboratories ${ }^{6,8,10}$ showing that consumption of fructose at levels comparable to that ingested by the upper 20th percentile of the human population combined with high salt intake results in elevated arterial pressure. Although the increase in arterial pressure is modest when assessed in conscious unstressed rats by telemetry (vs tail cuff where in there is the additional stress component of mild restraint), it is nonetheless reproducible, significant, and sustained. Moreover, the present data indicate that the elevation in arterial pressure is primarily due to a disproportionate rise in systolic pressure which may be more closely related to large artery stiffness (see below). The combination of high fructose and high salt intake has the potential to alter arterial wall cell function and structure independent of blood pressure. ${ }^{29,30}$ Hypertension, in turn, can influence vascular stiffness via cellular and structural responses to mechanical pressure-induced wall stress. ${ }^{31}$ Regardless of whether systolic hypertension is a cause or effect of arterial stiffness, ${ }^{31}$ the result is a complex interplay of factors that are influenced by consumption of fructose and high salt diet.

The observation that $4 \% \mathrm{NaCl}$ diet does not suppress PRA in either glucose-fed or fructose-fed animals is distinctly different than the classic suppression of PRA by high salt diets in rats provided with normal carbohydrate diets. $6,32,33$ Notably, earlier reports used higher salt diets (eg, $\geq 8 \% \mathrm{NaCl}$ ) that do not reflect typical human intake to inhibit PRA and did not report plasma Ang II levels. ${ }^{34,35}$

Table 4 Values for Plasma Glucose, PRA and Ang II and Renal Cortical Tissue Ang II After 3-Week Diet Regimen

\begin{tabular}{|l|c|c|c|c|c|}
\hline Diet & N & $\begin{array}{c}\text { Glucose } \\
\text { (mg/dL) }\end{array}$ & $\begin{array}{c}\text { PRA } \\
\text { (ng Angl per ml/hr) }\end{array}$ & $\begin{array}{c}\text { Plasma Ang II } \\
\text { (fmol/ml) }\end{array}$ & $\begin{array}{c}\text { Kidney Tissue Ang II } \\
\text { (fmol/g) }\end{array}$ \\
\hline Glu+NS & 8 & $132 \pm 2$ & $4.7 \pm 1.5$ & $34.2 \pm 4.6$ & $1,609 \pm 222$ \\
Glu+HS & 12 & $134 \pm 7$ & $5.6 \pm 2.8$ & $16.5 \pm 4.2^{*}$ & $1,829 \pm 269$ \\
Fru+NS & 8 & $130 \pm 5$ & $5.6 \pm 2.1$ & $38.5 \pm 5.8$ & $1,667 \pm 280$ \\
Fru+HS & 10 & $137 \pm 8$ & $6.7 \pm 2.8$ & $22.2 \pm 4.6^{\ddagger}$ & $1,403 \pm 223$ \\
Glu+NS+T & 10 & $138 \pm 6$ & $4.9 \pm 1.8$ & $16.0 \pm 2.3^{*}$ & $2,306 \pm 396$ \\
Glu+HS+T & 12 & $130 \pm 4$ & $5.1 \pm 3.5$ & $18.7 \pm 4.3$ & $1,376 \pm 168$ \\
Fru+NS+T & 9 & $142 \pm 7$ & $6.1 \pm 3.0$ & $25.4 \pm 5.1$ & $2,067 \pm 241$ \\
Fru+HS+T & 9 & $132 \pm 3$ & $5.3 \pm 3.3$ & $19.4 \pm 3.5$ & $1,509 \pm 290$ \\
\hline
\end{tabular}

Notes: Group definitions as in Table I. Values are mean \pm SE. $* P<0.05$ vs Glu+NS; $\ddagger P<0.05$ vs Fru+NS.

Abbreviations: PRA, plasma renin activity; Ang II, angiotensin II. 

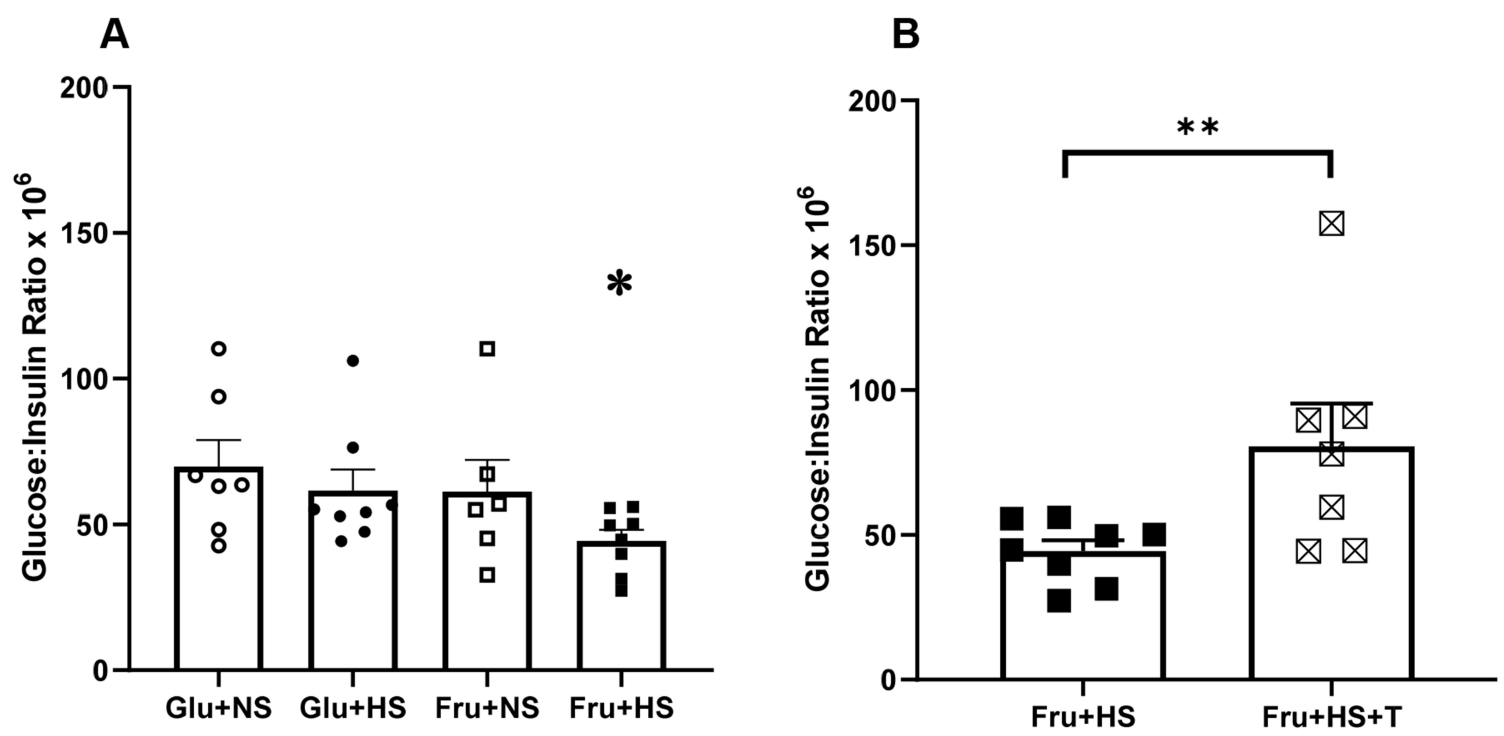

Figure 6 Glucose: insulin ratio $\left(\mathrm{nM} / \mathrm{nM} \times 10^{6}\right)$ in $(\mathbf{A})$ the four groups of rats and $(\mathbf{B})$ rats fed fructose-high salt alone or with Tempol $(+\mathrm{T})$. Group designations as in Figure 2. Values are mean $\pm \mathrm{SE} ; \mathrm{n}$ as indicated per group; $* \mathrm{P}<0.05$ vs other control groups; $* * P<0.05$ vs Fru+HS group.

Although high salt diet when ingested with fructose suppresses plasma Ang II but not renal tissue Ang II. The persistence of high intrarenal Ang II, which has been shown to increase proximal tubular sodium reabsorption, ${ }^{8,36}$ may very well be responsible for cumulative positive sodium balance and hypertension observed in this model. ${ }^{6}$ Furthermore, dietary fructose augments Ang IIstimulated superoxide formation by the kidney further enhancing proximal tubular sodium reabsorption and leading to volume-mediated hypertension. ${ }^{8,33,36}$ Since antioxidant with $+\mathrm{T}$ treatment does not influence either plasma or intrarenal Ang II levels, it is more likely that its ability to decrease hypertension relates, at least in part, to its ability to decrease oxidative stress directly on the vasculature. More definitive data measuring vascular ROS and its products, which were beyond the scope of the present study, will be required to address this issue directly.

\section{Left Ventricular Diastolic Dysfunction}

The axial heterogeneity of the aorta is well documented. ${ }^{37,38}$ For example, the ratio of elastic fibers to collagen changes from 3.1:1 in the ascending aorta to 2.8:1 in the thoracic aorta to $0.8: 1$ in the abdominal aorta. ${ }^{39}$ Attenuation of the elastic properties of the proximal ascending aorta can have profound influence on end-systolic cardiac wall stress due to increased afterload, one of the most important factors in developing cardiac hypertrophy, ${ }^{40}$ hence our choice to study the ascending aorta. In addition to the geometric properties of the left ventricle itself, aortic stiffness influences end-systolic cardiac wall stress. ${ }^{41}$ Although there is no evidence of left ventricular hypertrophy, our echocardiographic studies were performed after only three weeks on the respective diets. Nevertheless, the reduced $\mathrm{E} / \mathrm{A}$ ratio in the fructose-fed high salt rats reveals diastolic dysfunction which is the earliest indicator of hypertensive cardiac disease and precedes frank hypertrophy or systolic dysfunction. ${ }^{42}$ Importantly, the diastolic impairment is significant despite the arterial pressure lowering effect of isoflurane anesthesia during the scan. Furthermore, inhibition of oxidative stress does not ameliorate this diastolic dysfunction despite lower arterial pressure suggesting involvement of alternative mechanisms. Ingestion of fructose plus high salt even, in the short term, in the present study results in mildly elevated non-fasting blood glucose and a depressed glucose:insulin ratio consistent with insulin resistance similar to human pre-diabetes. Long-term studies have unequivocally demonstrated the development of left ventricular hypertrophy in metabolic syndrome; ${ }^{43}$ however, even prolonged pre-diabetes provides a constellation of physiological derangements that favor left ventricular remodeling in rats $^{44,45}$ and humans. ${ }^{46}$ In fact, fructose alone, albeit at a higher concentration (60\%), causes cardiac hypertrophy in mice after 10 weeks. ${ }^{47}$ Moreover, direct fructation of structural proteins $^{29}$ or alterations in sympathetic cardiac innvervation ${ }^{48}$ have been associated with diastolic dysfunction. Thus, despite the lack of frank left ventricular hypertrophy, the presence of a pre-diabetic state and insulin resistance is likely also contributing to the diastolic dysfunction in the fructose plus high salt group. ${ }^{49}$ 


\section{Impairment of Aortic and Renal Artery Compliance}

Diabetes $^{50,51}$ and hypertension ${ }^{52,53}$ are each independently associated with increased PWV. The increased PWV along with diminished radial ascending aortic strain and distensibility coefficient in fructose saltsensitive rats indicates that aortic compliance declines early with insulin resistance prior to overt diabetes. Greater aortic stiffness occurs with only modest (5-6 $\mathrm{mmHg}$ ) elevations in systolic (and mean) arterial pressure in the fructose-fed high salt rats. Increases in PWV are typically associated with greater pulse pressures. Although the change in pulse pressure was similar after three weeks of high salt, it is noteworthy that baseline pulse pressure in the fructose-fed high salt rats tended to be higher, albeit not statistically significant. Sympathetic innervation of the aorta is now recognized as a factor in aortic stiffening, ${ }^{54}$ and fructose-fed high salt rats display heightened renal sympathetic nerve activity. Renal denervation prevents the hypertension in this and other rodent models ${ }^{9,55}$ and ameliorates PWV in animal ${ }^{55}$ and human systolic hypertension. ${ }^{56}$ The contribution of cellular metabolic changes or ioninduced contractility cannot be totally excluded since triglycerides and cumulative salt balance are elevated in this model. ${ }^{6}$ Thus, aortic stiffness is only partially dependent on blood pressure and may also be influenced by biophysical changes in the structure and/or function of the arterial wall evoked by fructose plus high salt diet. Increased oxidative stress has been implicated in decreased aortic compliance in disease ${ }^{57}$ as well as normal aging. ${ }^{58,59}$ The agent, 4-hydroxy2,2,6,6-tetramethylpiperidine-1-oxyl, is a nonselective superoxide dismutase mimetic that reduces free radical formation and peroxynitrite formation, thereby limiting inflammation. Similar to findings with free radical scavenging in aging mice, ${ }^{60}$ our results indicate that attenuation of oxidative stress in fructose salt-sensitive rats rescues some aspects of elastic properties of the aorta, restoring normal PWV and radial ascending aortic strain.

Like the aorta, renal artery compliance is reduced which is reflected by the elevated RRI in fructose-fed high salt rats. In contrast to the ascending aorta, renal artery stiffness does not improve after treatment with this antioxidant. Although 4-hydroxy-2,2,6,6-tetramethylpiperidine- 1-oxyl has been shown to decrease renal superoxide anion and improve renal vascular resistance via a nitric oxide dependent mechanism, ${ }^{33,61}$ RRI tracks more closely with systemic vascular parameters, intrinsic renal artery compliance and pulsatility than with intrarenal vascular resistance. $^{20}$ This is not to say that this heightened pulsatility is not transmitted distally thereby ultimately resulting in hypertensive nephropathy and chronic kidney disease. While the present hemodynamic measurements have been performed in conscious, freely moving rats, ultrasonography necessitates isoflurane anesthesia. Importantly, isoflurane anesthesia does not alter measures of arterial stiffness. ${ }^{62}$

\section{Conclusion}

The rodent model used in these studies was chosen to mimic the amount of fructose and salt ingested by the upper fifth of the western population. Notably, impaired vascular compliance of the proximal ascending aorta and renal artery occurred after only short-term exposure to the fructose-high salt diet when insulin resistance but not frank hyperglycemia and only mild, but significantly elevated blood pressure ensued. The loss of vascular compliance results in transmission of the elevated pressure wave downstream and may, thereby, predispose to hypertensive organ damage including nephrosclerosis. The presence of left ventricular diastolic dysfunction with preserved systolic function strongly suggests that a diet of fructose combined with high salt exerts an independent effect on left ventricular relaxation. Importantly, anti-oxidant treatment normalizes fructoseinduced salt-sensitive hypertension and mitigates increase in aortic pulse wave velocity. These data, therefore, cannot distinguish whether aortic compliance is directly altered by reactive oxygen species induced by the high fructose plus high salt diet or indirectly via the elevation in arterial pressure or both. In contrast, left ventricular diastolic dysfunction and renal artery compliance are not altered by the free radical scavenger despite the decrease in blood pressure indicating that alternative mechanisms are responsible. Taken together, these findings indicate that a diet high in fructose and salt may lead to subtle, possibly progressive, pathophysiologic changes in the central cardiovascular system well before frank clinical disease is apparent. Changes in nutritional behavior and early assessment are imperative to foster cardiovascular health as we go forward. 


\section{Acknowledgments}

This work was funded by VA Merit Award RX000851 (NFR) and American Heart Association Transformational Project Award (18TPA34170169, CSC) whose support is gratefully acknowledged. PEL was supported by the Detroit Cardiovascular Training Program (NIH T32 HL120822). DK and PEL are co-first authors and contributed equally to this study. The authors would like to thank Ronald L. Thomas, PhD for his statistical expertise and Min Wu and Robert J. Pawlowicz for their assistance with technical and computer issues, respectively.

\section{Disclosure}

The authors report no conflicts of interest for this work.

\section{References}

1. Benjamin EJ, Muntner P, Alonso A, et al.; American Heart Association Council on E, Prevention Statistics C and Stroke Statistics S. Heart disease and stroke statistics- 2019 update: a report from the American Heart Association. Circulation. 2019;139:e56-e528.

2. Lewington S, Clarke R, Qizilbash N, Peto R, Collins R; Prospective Studies C. Age-specific relevance of usual blood pressure to vascular mortality: a meta- analysis of individual data for one million adults in 61 prospective studies. Lancet. 2002;360:1903-1913.

3. Tappy L, Le KA. Metabolic effects of fructose and the worldwide increase in obesity. Physiol Rev. 2010;90:23-46. doi:10.1152/ physrev.00019.2009

4. Jayalath VH, de Souza RJ, Ha V, et al. Sugar-sweetened beverage consumption and incident hypertension: a systematic review and meta-analysis of prospective cohorts. Am J Clin Nutr. 2015;102:914-921. doi:10.3945/ajcn.115.107243

5. Brown IJ, Stamler J, Van Horn L, et al. Sugar-sweetened beverage, sugar intake of individuals, and their blood pressure international study of macro/micronutrients and blood pressure. Hypertension. 2011;57:695-+. doi:10.1161/HYPERTENSIONAHA.110.165456

6. Gordish KL, Kassem KM, Ortiz PA, Beierwaltes WH. Moderate (20\%) fructose- enriched diet stimulates salt-sensitive hypertension with increased salt retention and decreased renal nitric oxide. Physiol Rep. 2017;5:e13162.

7. Palanisamy N, Venkataraman AC. Beneficial effect of genistein on lowering blood pressure and kidney toxicity in fructose-fed hypertensive rats. Br J Nutr. 2013;109:1806-1812.

8. Gonzalez-Vicente A, Hong NJ, Yang N, et al. Dietary fructose increases the sensitivity of proximal tubules to angiotensin II in rats fed high-salt diets. Nutrients. 2018;10:1244.

9. Soncrant T, Komnenov D, Beierwaltes WH, Chen H, Wu M, Rossi NF. Bilateral renal cryodenervation decreases arterial pressure and improves insulin sensitivity in fructose-fed Sprague-Dawley rats. Am J Physiol Regul Integr Comp Physiol. 2018;315:R529-R538.

10. Zenner ZP, Gordish KL, Beierwaltes WH. Free radical scavenging reverses fructose-induced salt-sensitive hypertension. Integr Blood Press Control. 2018;11:1-9. doi:10.2147/IBPC.S147674

11. Kopp UC, Dibona GF. Interaction between epinephrine and renal nerves in control of renin secretion rate. Am J Physiol. 1986;250: F999-F1007. doi:10.1152/ajprenal.1986.250.6.F999

12. Cooper LL, Rong J, Benjamin EJ, et al. Components of hemodynamic load and cardiovascular events: the Framingham Heart Study. Circulation. 2015;131:354-61; discussion 361. doi:10.1161/ CIRCULATIONAHA.114.011357
13. Kaess BM, Rong J, Larson MG, et al. Aortic stiffness, blood pressure progression, and incident hypertension. JAMA. 2012;308:875-881. doi:10.1001/2012.jama.10503

14. Cavalcante JL, Lima JA, Redheuil A, Al-Mallah MH. Aortic stiffness: current understanding and future directions. J Am Coll Cardiol. 2011;57:1511-1522. doi:10.1016/j.jacc.2010.12.017

15. Teixeira R, Vieira MJ, Goncalves A, Cardim N, Goncalves L. Ultrasonographic vascular mechanics to assess arterial stiffness: a review. Eur Heart $J$ Cardiovasc Imaging. 2016;17:233-246. doi:10.1093/ehjci/jev287

16. Ben-Shlomo Y, Spears M, Boustred C, et al. Aortic pulse wave velocity improves cardiovascular event prediction an individual participant meta-analysis of prospective observational data from 17,635 subjects. J Am Coll Cardiol. 2014;63:636-646. doi:10.1016/j. jacc.2013.09.063

17. Vlachopoulos C, Aznaouridis K, Stefanadis C. Prediction of cardiovascular events and all-cause mortality with arterial stiffness a systematic review and meta-analysis. $\mathrm{J}$ Am Coll Cardiol. 2010;55:1318-1327. doi:10.1016/j.jacc.2009.10.061

18. Bjallmark A, Lind B, Peolsson M, Shahgaldi K, Brodin LA, Nowak J. Ultrasonographic strain imaging is superior to conventional non-invasive measures of vascular stiffness in the detection of age-dependent differences in the mechanical properties of the common carotid artery. Eur $J$ Echocardiogr. 2010;11:630-636. doi:10.1093/ejechocard/jeq033

19. Bu Z, Ma J, Fan Y, et al. Ascending aortic strain analysis using 2-dimensional speckle tracking echocardiography improves the diagnostics for coronary artery stenosis in patients with suspected stable angina pectoris. $J$ Am Heart Assoc. 2018;7. doi:10.1161/ JAHA. 118.008802

20. O’Neill WC. Renal resistive index: a case of mistaken identity. Hypertension. 2014;64:915-917. doi:10.1161/HYPERTENSIONAHA. 114.04183

21. Kuznetsova T, Cauwenberghs N, Knez J, et al. Doppler indexes of left ventricular systolic and diastolic flow and central pulse pressure in relation to renal resistive index. $A m J$ Hypertens. 2015;28:535-545. doi:10.1093/ajh/hpu185

22. O'Rourke MF, Safar ME. Relationship between aortic stiffening and microvascular disease in brain and kidney - cause and logic of therapy. Hypertension. 2005;46:200-204. doi:10.1161/01.HYP.0000 168052.00426 .65

23. Morimoto A, Uzu T, Fujii T, et al. Sodium sensitivity and cardiovascular events in patients with essential hypertension. Lancet. 1997;350:1734-1737. doi:10.1016/S0140-6736(97)05189-1

24. Weinberger MH, Fineberg NS, Fineberg SE, Weinberger M. Salt sensitivity, pulse pressure, and death in normal and hypertensive humans. Hypertension. 2001;37:429-432. doi:10.1161/01.HYP.37.2.429

25. Coatney RW. Ultrasound imaging: principles and applications in rodent research. ILAR J. 2001;42:233-247. doi:10.1093/ ilar.42.3.233

26. Lindsey ML, Kassiri Z, Virag JAI, de Castro Bras LE, ScherrerCrosbie M. Guidelines for measuring cardiac physiology in mice. Am J Physiol Heart Circ Physiol. 2018;314:H733-H752. doi:10. 1152/ajpheart.00339.2017

27. Lee L, Cui JZ, Cua M, et al. Aortic and cardiac structure and function using high- resolution echocardiography and optical coherence tomography in a mouse model of marfan syndrome. PLoS One. 2016;11: e0164778. doi:10.1371/journal.pone.0164778

28. Rossi NF, Pajewski R, Chen H, Littrup PJ, Maliszewska-Scislo M. Hemodynamic and neural responses to renal denervation of the nerve to the clipped kidney by cryoablation in two-kidney, one-clip hypertensive rats. Am J Physiol Regul Integr Comp Physiol. 2016;310: R197-R208. doi:10.1152/ajpregu.00331.2015

29. Amani S, Fatima S. Glycation with fructose: the bitter side of nature's own sweetener. Curr Diabetes Rev. 2020;16. doi:10.2174/ 1389450121666200204115751 
30. Park KH, Jang W, Kim KY, Kim JR, Cho KH. Fructated apolipoprotein A-I showed severe structural modification and loss of beneficial functions in lipid-free and lipid-bound state with acceleration of atherosclerosis and senescence. Biochem Biophys Res Commun. 2010;392:295-300. doi:10.1016/j.bbrc.2009.12.179

31. Humphrey JD, Harrison DG, Figueroa CA, Lacolley P, Laurent S. Central artery stiffness in hypertension and aging: a problem with cause and consequence. Circ Res. 2016;118:379-381. doi:10.1161/ CIRCRESAHA.115.307722

32. Huang Y, Wang DH. Role of renin-angiotensin-aldosterone system in salt- sensitive hypertension induced by sensory denervation. $\mathrm{Am}$ J Physiol Heart Circ Physiol. 2001;281:H2143-9. doi:10.1152/ ajpheart.2001.281.5.H2143

33. Yang N, Gonzalez-Vicente A, Garvin JL. Angiotensin II-induced superoxide and decreased glutathione in proximal tubules: effect of dietary fructose. Am J Physiol Renal Physiol. 2020;318:F183-F192. doi:10.1152/ajprenal.00462.2019

34. Hayakawa Y, Aoyama T, Yokoyama C, et al. High salt intake damages the heart through activation of cardiac (pro) renin receptors even at an early stage of hypertension. PLoS One. 2015;10:e0120453. doi:10.1371/journal.pone.0120453

35. Chandramohan G, Bai Y, Norris K, Rodriguez-Iturbe B, Vaziri ND. Effects of dietary salt on intrarenal angiotensin system, $\mathrm{NAD}(\mathrm{P}) \mathrm{H}$ oxidase, COX-2, MCP-1 and PAI-1 expressions and NF-kappaB activity in salt-sensitive and -resistant rat kidneys. Am J Nephrol. 2008;28:158-167. doi:10.1159/000110021

36. Gonzalez-Vicente A, Cabral PD, Hong NJ, Asirwatham J, Saez F, Garvin JL. Fructose reabsorption by rat proximal tubules: role of $\mathrm{Na}$ $(+)$-linked cotransporters and the effect of dietary fructose. Am $J$ Physiol Renal Physiol. 2019;316:F473-F480. doi:10.1152/ ajprenal.00247.2018

37. Wilson JS, Taylor WR, Oshinski J. Assessment of the regional distribution of normalized circumferential strain in the thoracic and abdominal aorta using DENSE cardiovascular magnetic resonance. J Cardiovasc Magn Reson. 2019;21:59. doi:10.1186/s12968-0190565-0

38. Cox RH. Regional, species, and age related variations in the mechanical properties of arteries. Biorheology. 1979;16:85-94. doi:10.3233/ BIR-1979-161-212

39. Apter JT. Correlation of visco-elastic properties with microscopic structure of large arteries. IV. Thermal responses of collagen, elastin, smooth muscle, and intact arteries. Circ Res. 1967;21:901-918. doi:10.1161/01.RES.21.6.901

40. Vakili BA, Okin PM, Devereux RB. Prognostic implications of left ventricular hypertrophy. Am Heart J. 2001;141:334-341. doi:10.1067/mhj.2001.113218

41. Grossman W, Jones D, McLaurin LP. Wall stress and patterns of hypertrophy in the human left ventricle. $J$ Clin Invest. 1975;56:56-64. doi:10.1172/JCI108079

42. Sorrentino MJ. The evolution from hypertension to heart failure. Heart Fail Clin. 2019;15:447-453. doi:10.1016/j.hfc.2019.06.005

43. Jorgensen PG, Jensen MT, Biering-Sorensen T, et al. Burden of uncontrolled metabolic risk factors and left ventricular structure and function in patients with type 2 diabetes mellitus. $J$ Am Heart Assoc. 2018;7:e008856. doi:10.1161/JAHA.118.008856

44. D'Souza A, Howarth FC, Yanni J, et al. Left ventricle structural remodelling in the prediabetic Goto-Kakizaki rat. Exp Physiol. 2011;96:875-888. doi:10.1113/expphysiol.2011.058271

45. Mizushige K, Yao L, Noma T, et al. Alteration in left ventricular diastolic filling and accumulation of myocardial collagen at insulin-resistant prediabetic stage of a type II diabetic rat model. Circulation. 2000;101:899-907. doi:10.1161/01.CIR.101.8.899
46. Rospleszcz S, Schafnitzel A, Koenig W, et al. Association of glycemic status and segmental left ventricular wall thickness in subjects without prior cardiovascular disease: a cross-sectional study. BMC Cardiovasc Disord. 2018;18:162. doi:10.1186/s12872-018-0900-7

47. Park JH, Ku HJ, Kim JK, Park JW, Lee JH. Amelioration of high fructose- induced cardiac hypertrophy by Naringin. Sci Rep. 2018;8:9464. doi:10.1038/s41598-018-27788-1

48. Liu Y, Li B, Li M, Yu Y, Wang Z, Chen S. Improvement of cardiac dysfunction by bilateral surgical renal denervation in animals with diabetes induced by high fructose and high fat diet. Diabetes Res Clin Pract. 2016;115:140-149. doi:10.1016/j.diabres.2015.12.012

49. Waddingham MT, Sonobe T, Tsuchimochi H, et al. Diastolic dysfunction is initiated by cardiomyocyte impairment ahead of endothelial dysfunction due to increased oxidative stress and inflammation in an experimental prediabetes model. $J$ Mol Cell Cardiol. 2019;137:119-131. doi:10.1016/j.yjmcc.2019.10.005

50. Agnoletti D, Mansour AS, Zhang Y, et al. Clinical interaction between diabetes duration and aortic stiffness in type 2 diabetes mellitus. J Hum Hypertens. 2017;31:189-194. doi:10.1038/jhh.2016.58

51. Rahman S, Ismail AA, Ismail SB, Naing NN, Rahman AR. Early manifestation of macrovasculopathy in newly diagnosed never treated type II diabetic patients with no traditional CVD risk factors. Diabetes Res Clin Pract. 2008;80:253-258. doi:10.1016/j. diabres.2007.12.010

52. Guers JJ, Farquhar WB, Edwards DG, Lennon SL. Voluntary wheel running attenuates salt-induced vascular stiffness independent of blood pressure. Am J Hypertens. 2019;32:1162-1169. doi:10.1093/ ajh/hpz128

53. Armstrong MK, Schultz MG, Picone DS, Sharman JE. Aortic-tobrachial artery stiffness gradient is not blood pressure independent. J Hum Hypertens. 2019;33:385-392.

54. Holwerda SW, Luehrs RE, DuBose L, et al. Elevated muscle sympathetic nerve activity contributes to central artery stiffness in young and middle-age/older adults. Hypertension. 2019;73:1025-1035.

55. Hohl M, Linz D, Fries P, et al. Modulation of the sympathetic nervous system by renal denervation prevents reduction of aortic distensibility in atherosclerosis prone ApoE-deficient rats. $J$ Transl Med. 2016;14:167.

56. Fengler K, Rommel KP, Hoellriegel R, et al. Pulse wave velocity predicts response to renal denervation in isolated systolic hypertension. J Am Heart Assoc. 2017;6:e005879.

57. Kals J, Kampus P, Kals M, et al. Inflammation and oxidative stress are associated differently with endothelial function and arterial stiffness in healthy subjects and in patients with atherosclerosis. Scand J Clin Lab Invest. 2008;68:594-601.

58. Zhou RH, Vendrov AE, Tchivilev I, et al. Mitochondrial oxidative stress in aortic stiffening with age: the role of smooth muscle cell function. Arterioscler Thromb Vasc Biol. 2012;32:745-755.

59. Canugovi $\mathrm{C}$, Stevenson MD, Vendrov $\mathrm{AE}$, et al. Increased mitochondrial NADPH oxidase 4 (NOX4) expression in aging is a causative factor in aortic stiffening. Redox Biol. 2019;26:101288.

60. de Picciotto NE, Gano LB, Johnson LC, et al. Nicotinamide mononucleotide supplementation reverses vascular dysfunction and oxidative stress with aging in mice. Aging Cell. 2016;15:522-530.

61. Patterson ME, Mouton CR, Mullins JJ, Mitchell KD. Interactive effects of superoxide anion and nitric oxide on blood pressure and renal hemodynamics in transgenic rats with inducible malignant hypertension. Am J Physiol Renal Physiol. 2005;289:F754-9.

62. Morgan EE, Casabianca AB, Khouri SJ, Kalinoski AL. In vivo assessment of arterial stiffness in the isoflurane anesthetized spontaneously hypertensive rat. Cardiovasc Ultrasound. 2014;12:37. 


\section{Publish your work in this journal}

Integrated Blood Pressure Control is an international, peer-reviewed open-access journal focusing on the integrated approach to managing hypertension and risk reduction. Treating the patient and comorbidities together with diet and lifestyle modification and optimizing healthcare resources through a multidisciplinary team approach constitute key features of the journal. This journal is indexed on
American Chemical Society's Chemical Abstracts Service (CAS) The manuscript management system is completely online and includes a very quick and fair peer-review system, which is al easy to use. Visit http://www.dovepress.com/testimonials.php to read real quotes from published authors. 